\title{
SÍNDROME INFLAMATORIO SISTÉMICO ASOCIADO A COVID-19 Y LA ENFERMEDAD DE KAWASAKI. UNA REVISIÓN BIBLIOGRÁFICA
}

\section{Systemic Inflammatory Syndrome associated with Covid-19 and Kawasaki Disease. A bibliographic review}

\section{Elaine Priscilla Pozo y Paul Guerra Rosadob}

Recibido: 25 de noviembre, $2020 \bullet$ Aprobado: 18 de enero, 2021

Cómo citar: Pozo EP, Rosado PG. Síndrome inflamatorio sistémico asociado a Covid-19 y la enfermedad de Kawasaki. Una revisión bibliográfica. cysa [Internet]. 12 de octubre de 2021 [citado 15 de octubre de 2021];5(3):101-6. Disponible en: https://revistas.intec. edu.do/index.php/cisa/article/view/2303

\section{Resumen}

Introducción: la enfermedad de Kawasaki (EK) se define como una vasculitis multisistémica aguda y autolimitada asociada a lesiones coronarias. Los primeros casos se observaron en Japón a finales de 1960 y recibió dicho nombre debido al médico japonés que la describió por primera vez en el ańo 1967, Tomisaku Kawasaki.

El síndrome inflamatorio multisistémico (SIM-C) se define como una patología que afecta generalmente a niños y adolescentes de 0 a 19 ańos, caracterizada por presencia de fiebre desde hace tres días o más y dos de los criterios diagnósticos establecidos.

Objetivo: determinar y establecer las asociaciones clínicas entre el síndrome inflamatorio multisistémico asociado a Covid-19 y la enfermedad de Kawasaki.

Metodología: estudio comparativo e informativo, en donde se analizaron 16 fuentes bibliográficas para establecer asociaciones entre el síndrome inflamatorio multisistémico vinculado a Covid-19 y la enfermedad de Kawasaki.

\footnotetext{
a Hospital Infantil Dr. Robert Reid Cabral, Santo Domingo, República Dominicana.Correo-e: elaine.pozo@intec.edu.do
}

\begin{abstract}
Introduction: Kawasaki disease (KD) is defined as an acute and self-limiting multisystemic vasculitis associated with coronary lesions. The first cases were observed in Japan in the late 1960s and it was named after the Japanese doctor who first described it in 1967, Tomisaku Kawasaki.

The multisystemic inflammatory syndrome (SIM-C) is defined as a pathology that generally affects children and adolescents from 0 to 19 years of age, characterized by the presence of fever for three days or more and two of the established diagnostic criteria.

Objective: Determine and establish the clinical associations between covid-19 associated multisystemic inflammatory syndrome and kawasaki disease.

Methodology: Comparative and informative study, where 16 bibliographic sources were analyzed to establish associations between the multisystemic inflammatory syndrome associated with covid-19 and kawasaki disease.
\end{abstract}

\footnotetext{
b Estudiante de Medicina del Instituto Tecnológico de Santo Domingo (INTEC). Correo-e: paulguerra11@hotmail.com
} 
Conclusión: la enfermedad de Kawasaki y el síndrome inflamatorio multisistémico poseen similitud en cuanto a manifestaciones clínicas se refiere, así como también, de acuerdo a las hipótesis, podrían estar explicadas por una infección viral como desencadenante de la enfermedad; ambas presentan afectación a nivel ocular, gastrointestinal y cardíaca.

Palabras clave: enfermedad de Kawasaki; aneurisma coronario; vasculitis; infección por el SARS-COV-2 (n COV), neumopatía infecciosa.

\section{Introducción}

La enfermedad de Kawasaki (EK) se define como una vasculitis multisistémica aguda y autolimitada asociada a lesiones coronarias ${ }^{1}$. Los primeros casos se observaron en Japón a finales de 1960 y recibió dicho nombre debido al médico japonés que la describió por primera vez en el año 1967, Tomisaku Kawasaki².

La etiología de la EK todavía se desconoce, aunque sus características clínicas, analíticas y epidemiológicas sugieren un origen o desencadenante infeccioso $^{3}$. De igual forma, es bien conocido el hecho de que la EK es la causa más común de enfermedad cardíaca adquirida, que por lo general se desarrolla antes de los cinco años de edad ${ }^{1}$.

Por otra parte, la Organización Mundial de la Salud publicó un informe científico el 15 de mayo de 2020, en donde especificaba el hecho de que recientemente se han descrito grupos de nińos y adolescentes en Europa y en Norteamérica que han sido ingresados en Unidad de Cuidados Intensivos (UCI), en vista a un cuadro inflamatorio multisistémico de características similares a la EK y al síndrome de choque tóxico (ST estreptococico) $)^{2}$. Las hipótesis iniciales, basadas en pruebas de laboratorio, indicaban que dicha situación podría estar relacionada con COVID-194.

Con respecto a los casos referidos, el 7 de abril se notificó en los Estados Unidos un caso de una niña
Conclusions: Kawasaki disease and multisystemic inflammatory Syndrome have similarities in terms of clinical manifestations, as well as, according to the hypotheses, they could be explained by a viral infection as a trigger for the disease; both present affectation at the ocular, gastrointestinal and cardiac levels.

Keywords: Kawasaki disease; coronary aneurysm; vasculitis; SARS-CoV-2 (n COV) infection; infectious lung disease.

de 6 meses de edad con EK clásico y COVID-19 concurrente, y el 27 de abril de 2020 las autoridades de salud del Reino Unido informaron de un número de niños gravemente enfermos que presentaban signos de shock tóxico, y algunos positivos a COVID-19 concomitantemente; y es que estos pacientes cursaban con fiebre persistente, estado hiperinflamatorio y algunos, afectación cardíacas .

En vista a las consideraciones establecidas el objetivo general de este trabajo es determinar y establecer las asociaciones clínicas entre el síndrome inflamatorio multisistémico asociado a Covid-19 y la enfermedad de Kawasaki, mediante una revisión bibliográfica de publicaciones realizadas.

\section{¿Existe algún tipo de relación con respecto a la etiología de la EK y el síndrome inflamatorio multisistémico?}

En un estudio realizado en junio de 2018 por la Asociación Española de Pediatría, cuyo propósito era describir la presencia de infecciones en los niños con EK, se expuso que algunos estudios describen que hasta la mitad de los casos de EK tienen uno o más virus respiratorios detectados por PCR, incluso refieren un estudio realizado por Chang et al, en donde una de las conclusiones especificaba el hecho de que las infecciones víricas se asocian a $\mathrm{EK}^{1}$.

La etiología de la EK todavía se desconoce, aunque se piensa en un origen o desencadenante infeccioso $^{3,6}$, y es que incluso, en las hipótesis con 
relación a la patogenia de dicha enfermedad, se sugiere la presencia de un patógeno que ingresa por las vías aéreas superiores y posteriormente penetra a través del epitelio bronquial, donde es captado por los macrófagos tisulares, que desencadena luego la respuesta inmune innata ${ }^{7,8}$. Otra hipótesis sugiere la existencia de una respuesta inmune de IgA oligoclonal en lugar de una policlonal ${ }^{7}$. De modo que la etiopatogenia de la EK aún no está del todo clara, pero actualmente se discute desde el punto de vista de la interacción de dos aspectos, la susceptibilidad genética y la infección?

Con respecto al síndrome inflamatorio multisistémico (SIM-C), las hipótesis iniciales, según la OMS, apuntan a que este síndrome puede estar relacionado a COVID-194, pero aún no se ha determinado una etiología específica. Sin embargo, observando los hallazgos actuales podría relacionarse, al igual que la $\mathrm{EK}$, a una posible infección por SARS-CoV-2.

\section{Criterios diagnósticos}

Se ha observado que la EK y SIM-C comparten síntomas comunes como fiebre de larga duración, erupción cutánea, linfadenopatía, diarrea y elevación de biomarcadores inflamatorios, pero a su vez el SIM presenta algunas características distintas, como un inicio más antiguo de las manifestaciones clínicas, prevalencia de síntomas abdominales y más casos con disfunción sistólica del ventrículo derecho e insuficiencia cardíaca aguda ${ }^{9}$. En vista de ello se cree que la comprensión de la EK podría ayudar en el análisis del SIM-C ${ }^{9}$, a pesar de que existen criterios diagnósticos diferentes entre una y otra.

La definición preliminar del SIM-C es niños y adolescentes de 0 a 19 años con fiebre prolongada y dos de los criterios siguientes ${ }^{4}$ :

a) erupción cutánea o conjuntivitis no purulenta bilateral o signos de inflamación mucocutánea en boca, manos o pies, como son pápulas eritematosas;

b) hipotensión o choque; c) características de disfunción miocárdica, pericarditis, valvulitis o anomalías coronarias (incluidos signos ecocardiográficos o valores elevados de troponina/NT-proBNP);

d) pruebas de coagulopatía alteradas (a partir del TP, el TTPA o de valores elevados de dímeros D);

e) problemas gastrointestinales agudos (diarrea, vómitos o dolor abdominal).

f) Con respecto a los criterios diagnósticos de la $\mathrm{EK}$, son los especificados en la siguiente tabla:

Tabla 1. Criterios diagnósticos de la enfermedad de Kawasaki

El diagnóstico se considera confinado cuando a la fiebre de cinco dias o más de evolución, se asocian cuatro de los cinco criterios clinicos siguientes y la enfermedad no puede ser explicada por otra etiologia conocida.

1. Inyección conjuntival bulbar sin exudado.

2. Cambios en la mucosa bucal como labios agrietados y rojos lengua aframbuesada y faringe eritematosa.

3. Erupción eritematosa generalizada polimorfa que puede ser morbiliforme, maculopapular, escarlatini forme, urticanana o lino critema multiforme.

4. Cambios en las extremidades: induración y enrojccimiento de palmas y plantas, descamación periungueal de los dedos de manos y pies aproximadamente a las dos semanas de iniciado el cuadro.

5. Adenopatia cervical solitaria mavor de $1,5 \mathrm{~cm}$.

Olorido de Pediatrios 109; 104(1).

Fuente: archivos de Pediatría del Uruguay, Revista Scielo ${ }^{17}$.

Como se aprecia, claramente, existe alguna similitud en los criterios a evaluar entre ambas patologías, como son la erupción cutánea y la conjuntivitis. En ese orden siempre debe considerarse el hecho de que existen tipos de EK, las cuales son EK completa, incompleta y atípica ${ }^{3}$, en vista a los criterios que se cumplen y a los que no se observan en el paciente, o a algún tipo de sintomatología no incluida dentro de los criterios de la EK clásica. 


\section{Manifestaciones oculares}

La Revista Mexicana de Oftalmología publicó un artículo en el año 2015 en donde observaron que en un total de 96 pacientes con EK que fueron valorados por el servicio de oftalmología, el $17.7 \%$ presentó complicaciones oculares como la hiperemia conjuntival, uveítis anterior, queratitis, alteraciones de la retina y papilitis ${ }^{2}$. Los mismos concluyeron que la manifestación ocular de la EK más común fue la inyección conjuntival, de igual forma especificaron que las manifestaciones oculares se presentan en su mayoría en la fase aguda, más comunes en hombres y en menores de cinco años ${ }^{2}$.

En el caso del SIM, es muy frecuente la conjuntivitis no purulenta bilateral, tanto así que ha sido incluida como parte de los criterios diagnósticos ${ }^{4}$, lo cual es una similitud más con respecto a la EK.

\section{Manifestaciones gastrointestinales}

Dentro de los criterios diagnósticos del SIM se pueden observar problemas gastrointestinales agudos como son la diarrea, vómitos y dolor abdominal ${ }^{2}$, pero no sucede lo mismo en los criterios especificados con respecto a la EK clásica. Ahora bien, a pesar de que no está especificado dentro de los criterios, los niños que cursan con EK también tienden a presentar manifestaciones gastrointestinales como son la diarrea y los vómitos ${ }^{3}$, al igual que en el SIM.

Entre el mes de febrero y abril de 2020, la Revista Elsevier España publicó un artículo en donde hizo énfasis en la infección por COVID-19 en pacientes con enfermedad inflamatoria intestinal, y esto debido a que dichos pacientes tienden a acudir con mayor frecuencia a diferentes centros sanitarios, lo que puede incrementar el riesgo de contagio ${ }^{11}$. El SIM se ha relacionado con la infección por COVID-194, lo que nos hace suponer que tal vez los criterios especificados en el SIM, con respecto a los problemas gastrointestinales, podrían de igual forma tener una explicación desde el punto de vista de la patogenia de la infección por SARS-COV-2.

\section{Afectación cardíaca}

En el 2020, Journal of the Formosan Medical Association publicó un artículo en donde especificaron que los nińos con SIM (enfermedad similar a Kawasaki), a diferencia de aquellos con EK, tenían más signos de falla cardíaca acompañada de hipotensión y de activación de macrófagos ${ }^{10}$.

Con respecto al SIM, la mayoría de los casos en donde se evidencia afectación cardíaca, lo que se observa por lo regular es disfunción sistólica del ventrículo derecho e insuficiencia cardíaca aguda'. En el caso de la EK, es muy frecuente la aparición de aneurismas coronarios, incluso en un estudio publicado por Sánchez et al., en el año 2018, informa que se diagnosticaron 144 casos de niños que presentaban alteración coronaria, 69 casos cursaban con aneurismas coronarios y 5 casos con aneurismas coronarios gigantes ${ }^{12}$. De igual forma resaltan que el porcentaje de aneurismas gigantes en la serie KawaRace (red pediátrica de ámbito nacional para el estudio de EK), es muy alto ${ }^{12}$.

\section{COVID-19 y síndrome inflamatorio multisis- témico (SIM-C)}

La enfermedad por el nuevo coronavirus SARS-CoV-2 apareció en Wuhan, China, a finales del año 2019, y los primeros trabajos publicados con análisis pediátrico procedentes de dicho país describen cursos clínicos más leves que en adultos, fundamentalmente por sintomatología respiratoria ${ }^{13}$. Las razones descritas que apoyan esta idea se basan en que en pacientes pediátricos hay una alta población de linfocitos $\mathrm{T}$ vírgenes, los receptores de la enzima convertidora de angiotensina 2 (ACE2) son diferentes a los del adulto, y debido también a que estos, por lo regular, no cursan con comorbilidades ${ }^{9,14}$.

El SIM se observó especialmente en niños en el Reino Unido, los cuales presentaban signos de shock y manifestaciones similares a la $\mathrm{EK}$, pero que no era EK ni Shock Tóxico ${ }^{5}$, sino una nueva patología que 
se ha relacionado a la infección por COVID-19, por aparecer posterior a la infección por SARS-CoV-2. Al mismo tiempo, la lesión miocárdica causada por la infección por coronavirus es uno de los principales factores que contribuyen al mal pronóstico ${ }^{15,16}$.

Actualmente, el SIM aún es estudiado, en la búsqueda de la etiología y patogenia específica que ilustre un poco más al personal de salud sobre cómo tratarla.

\section{Conclusión}

La enfermedad de Kawasaki y el síndrome inflamatorio multisistémico poseen similitud en cuanto a manifestaciones clínicas se refiere, como lo son la conjuntivitis no purulenta, alteraciones gastrointestinales, erupción cutánea y alteración cardíaca. De acuerdo con las hipótesis ambas enfermedades podrían estar explicadas por una infección viral como desencadenante de la enfermedad.

La EK y el SIM presentan afectación a nivel ocular, siendo lo más frecuente la conjuntivitis bilateral no purulenta; alteraciones gastrointestinales como diarrea y náuseas, y alteraciones cardíacas, con mayor prevalencia en el SIM la disfunción sistólica del ventrículo derecho, a diferencia de la EK, en donde lo más predominante son los aneurismas coronarios.

\section{Bibliografía}

1. Fernández-Cooke E, Tascón A, Antón-López J, Lozano G, Daniel C, Sánchez-Manubens J, Calvo, Cristian. Infecciones previas o coincidentes con la sospecha de enfermedad de Kawasaki ¿debemos cambiar nuestra actitud? An Pediatric (Barc). 2019;90(4):213-8.

2. Cabello A, Belina Á, Hortencia F, Carlos J. Manifestaciones oculares en enfermedad de Kawasaki. Experiencia en el Instituto Nacional de Pediatría. Rev Mex Oftalmol. 2016;90(1):3-8.
3. Sánchez-Manubens J, Antón J, Rosa B, Estibaliz I, Calzada-Hernandez J, Rodó X, Josep-Antón M, y el Grupo de Trabajo en Enfermedad de Kawasaki en Cataluña. En Cataluña la Enfermedad de Kawasaki es más prevalente en las zonas rurales. An Pediatric (Barc). 2017;87(4): 226-31.

4. Organización Mundial de la Salud. Síndrome inflamatorio multisistémico en niños y adolescentes con COVID-19. 2020. [En línea] Disponible en: WHO/2019-nCoV/Sci_Brief/ Multisystem_Syndrome_Children/2020.1 [Actualizada 2020].

5. Ministerio de Salud Pública del Ecuador. Alerta Epidemiológica por Síndrome Inflamatorio multisistémico (SIM-C) en niños y adolescentes (menores de 19 ańos), temporalmente relacionado con COVID-19. Rev Elsevier. [31 de mayo de 2020]

6. Tascóna A, Centeno F, Rojo H, Fernández-Cookee E, Sánchez-Manubensg J, Pérez-Lescure J. Consenso nacional sobre diagnóstico, tratamiento y seguimiento cardiológico de la enfermedad de Kawasaki. An Pediatric (Barc). 2018;89(3):188.e1-188.e22.

7. Mejía S, Correa C, Suárez C, Quiñonez M, Lobo R. Enfermedad de Kawasaki. Rev Colomb Cardiol. 2017;24(3):307.e1- 307.e6.

8. Rabadán A, Recio A, Garcíab I, Rupérezc E. Detección precoz y manejo de la enfermedad de Kawasaki: la inadvertida enfermedad de Kawasaki incompleta. Rev Clin Med Fam 2012;5 (3):212-5.

9. Ebina-Shibuya R, Namkoong H, Shibuya $Y$, Horita N. Multisystem Inflammatory Syndrome in Children (MIS-C) with COVID-19: Insights from simultaneous familial Kawasaki Disease cases, International Journal of Infectious Diseases. 2020; Available from: https://doi.org/10.1016/j. ijid.2020.06.014 
10. Alizargar J. The novel coronavirus (COVID19) and the risk of Kawasaki disease in children, Journal of the Formosan Medical Association. November 2020;119(11):1713-4. Available from https://doi.org/10.1016/j.jfma.2020.05.030

11. Martín-Jiménez I, et al. Covid-19 y enfermedad inflamatoria intestinal: preguntas surgidas de la atención y seguimiento de los pacientes durante la fase inicial de la pandemia (febrero-abril 2020). Gastroenterol Hepatol. 2020;43(7):40813. Disponible en: https://doi.org/10.1016/j. gastrohep.2020.05.003

12. Sanchez A, et al. Aneurismas coronarios gigantes en lactantes con enfermedad de kawasaki. An pediatric (Barc). 2018;88(3):178-80.

13. Chacón-Aguilar R, et al. COVID-19: síndrome febrily clínica neurológica en neonato. An Pediatric (Barc). 2020;92(6):373-4. Disponible en: https://doi.org/10.1016/j.anpedi.2020.04.012

14. Aguilar-Shea A, Gallardo-Mayo C. Covid-19 y la esperanza de la inmunidad. Aten Primaria. 2020;52(8):593-4. Disponible en: https://doi. org/10.1016/j.aprim.2020.05.004
15. Mengmeng Z, Menglong W, Jishou Z, Jing Y, Yao X, Zhen W, Di Y, Jianfang L, Jun W. Advances in the relationship between coronavirus infection and $\mathrm{T}$ cardiovascular diseases. Biomedicine \& Pharmacotherapy, 2020;127:110230.

16. Antona C, Rivera R, Huertas M, Fernández C, Pignatelli R. Comunicación: Alerta sobre asociación COVID 19 y probable Enfermedad de Kawasaki en pacientes pediátricos. [10 de mayo de 2020] Disponible en: http://www. siacardio.com/novedades/covid-19/alerta-sobre-asociacion-covid-19-y-probable-enfermedad-de-kawasaki-en-pacientes-pediatricos/

17. Belén M, García M, Martínez L. Enfermedad de Kawasaki en un lactante de dos meses. Revista Scielo. 2003;74(3). Disponible en: http://www. scielo.edu.uy/scielo.php?script=sci_arttext\&pid=S1688-12492003000300009 